\title{
Organic Semiconductivity and Photovoltaism: Concepts and applications
}

\author{
Soufiyan Hamham *, Abdelouahed Cherqaoui, Said Belaaouad and Youssef Naimi * \\ Laboratory of Physical Chemistry of Materials, Faculty of Sciences Ben M'sik, Hassan II University of \\ Casablanca, Morocco
}

\begin{abstract}
This work has for the objective, the theoretical explanation of the semi-conduction mechanisms as well as the photovoltaism in molecular organic materials by the creation of excitons and the transfer of $\pi$ electrons of a donor (P3HT) to an acceptor (PCBM). In focusing on the broad technological applications of the molecular optoelectronic phenomena, this will provide the capital importance of the molecular electronic as a scientific and technical revolution which extends from the energy, has medicine up to the areas confusing nanotechnology and quantum electronics.
\end{abstract}

Keywords: Organic Photovoltaism; organic semi-conductivity; Donor-Acceptor interface; the HOMO-LUMO transition; vibration-orbit coupling.

\section{Introduction}

The world of semiconductors is dominated, in market terms, by the silicon. The latter has ad bandgap of $1.11 \mathrm{eV}$ and has been one of the first materials used (with the germanium) for the manufacture of electronic components ${ }^{1}$. In 1960, a new family appeared, that of III-V materials (GaAs, InP, GaN, etc.) ${ }^{2}$. The components manufactured from these semiconductors, which have remarkable physical properties, play a significant role in the microwave applications in electric power, but especially in the area of optoelectronics ${ }^{3}$.

The ternary alloys of the III-V compounds semiconductors are considered as an essential class of materials, and this thanks to the adjustment of their parameter of the network, energy gap, refractive index, optical constants and other physical parameters that can be adjusted and controlled for the obtaining of component optoelectronics desired excellent ${ }^{4,5}$.

The energy gap, optical constants, and dielectric permittivity are essential parameters and play an essential role in physic. A precise knowledge of the structure of bands of the material studied is necessary for the evaluation of its field of application, transitions, direct and indirect; in the function of the composition are particularly of significant importance for the study and the manufacture of solar cells ${ }^{6}$.

The ternary alloys semi-conductors of the III-V compounds of the form $\mathrm{ABxC} 1-\mathrm{X}$ where (x) represents $\mathrm{AB}$ compound concentration and $(1-\mathrm{x})$ the

*Corresponding author: Soufiyan Hamham

Email address: soufiyanhamham01@gmail.com

DOI: http://dx.doi.org/10.13171/mjc91190820600sh
AC compound concentration have attracted considerable attention for optoelectronic devices applications thanks to the adjustment of several of their physical parameters as a function of the molar fraction $\mathrm{x}^{5,7,8}$.

\subsection{Crystal structure of semiconductors}

A crystal may be represented from a basic cell which is repeated periodically, thus forming the crystal lattice. According to the nature of the operations of symmetry which leave the crystal structure invariant, one is led to set seven crystalline systems, among which the cubic system ${ }^{5,9}$.

\subsubsection{Simple Semiconductor}

The simple semiconductors Tell that if, Ge, C Diamond, crystallize in a cubic structure with centered faces ${ }^{10}$ (Figure 1 and 2).

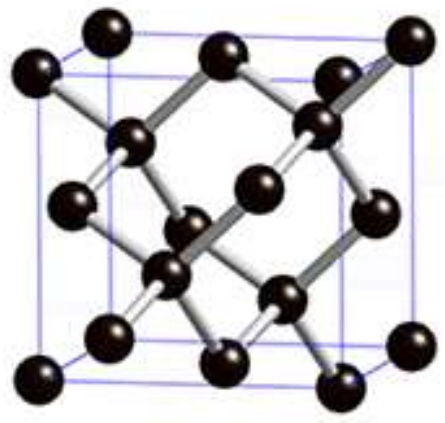

Figure 1. crystal structure diamond

Received July 5, 2019 Accepted July 24, 2019 Published August 20, 2019 


\subsubsection{Compound semiconductor}

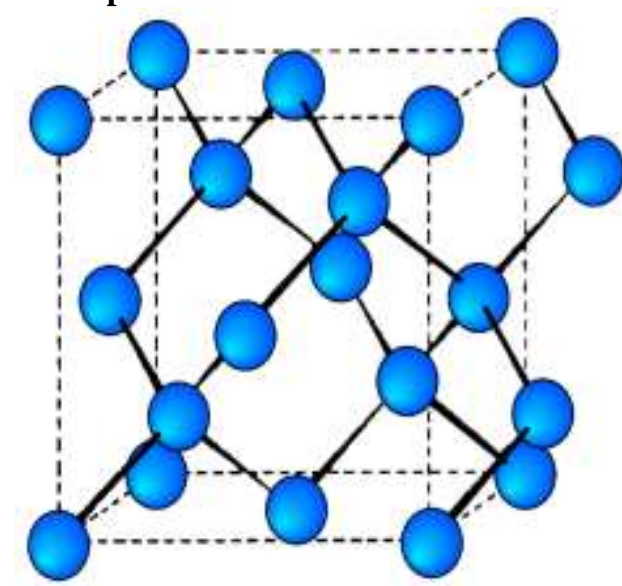

Figure 2. crystal structure wörtzite

\subsection{The different types of semiconductors}

\subsubsection{Intrinsic Semiconductor}

A semiconductor is intrinsic said when the crystal is not polluted (voluntarily or not) by impurities that can change the concentration of free charge carriers ${ }^{11}$.

Has the thermal equilibrium, total densities of electrons in the conduction band and holes in the valence band are data by the following expressions ${ }^{12}$ :

$n_{e}=N_{c} e^{\left[\frac{E_{F}-E_{C}}{k T}\right]}$ With $N_{c}=\frac{2}{h^{3}}\left(2 \pi m_{e}^{*} k T\right)^{\frac{3}{2}}$

$n_{h}=N_{v} e^{\left[\frac{E_{v}-E_{F}}{k T}\right]} \quad$ With $\quad N_{v}=\frac{2}{h^{3}}\left(2 \pi m_{h}^{*} k T\right)^{\frac{3}{2}}$

Where: $N_{c}$ and $N_{v}$ are respectively the effective density of states of electrons in the conduction band and the effective density the holes of states in the valence band ${ }^{13,14}$.

$\boldsymbol{m}_{\boldsymbol{e}}^{*}$ and $\boldsymbol{m}_{\boldsymbol{h}}^{*}$ are respectively the active masses of the electron and hole

$E_{v}$ is the highest level of the valence band energy;

$E_{C}$ Is the lowest level of the conduction band energy;

$E_{F}$ The Fermi energy of the material;

$\mathrm{h}$ is the Planck constant;

$\mathrm{k}$ is the Boltzmann's constant;

$\mathrm{T}$ is the temperature in $\mathrm{K}$.

A semiconductor is an intrinsic material devoid of any impurity likely to change the density of the charge carriers. The electrons in the conduction band could not be that resulting from the thermal excitation of the valence band electrons. The holes and electrons exist necessarily by pairs $\left(n_{i}=n_{e}=n_{h}\right)$, so one obtains the following expressions for the intrinsic concentration of carriers and the level of intrinsic Fermi ${ }^{15,16,17}$ :

$n_{i}=\left(N_{c}-N_{v}\right)^{1 / 2} \exp \left[-\frac{E_{g}}{2 k T}\right]$

$E_{F}=\frac{1}{2}\left[E_{v}+E_{c}\right]+\frac{3}{4} k T \log \left(\frac{m_{h}^{*}}{m_{e}^{*}}\right)$

\subsubsection{Intrinsic temperature}

The transition between the exhaustion regime and the intrinsic regime mark the temperature limit for a semiconductor device, called intrinsic temperature. Hence the importance, in the case of applications in high temperature, to push to the maximum this transition if one defines the intrinsic temperature $T_{i}$ as the temperature where the intrinsic concentration $n_{i}\left(T_{i}\right)$ becomes comparable or equal to the concentration of the impurities net doping ${ }^{9}$

$n_{i}\left(T_{i}\right)=\left|N_{d}-N_{a}\right|$

\subsubsection{Extrinsic Semiconductor}

The introduction of certain impurities in a semiconductor material allows to change the number of free charge carriers, to choose the type of conduction (by electron or by holes) and check the conductivity ${ }^{18}$.

\subsection{4. n Doped semiconductor}

The atoms have five valences such as arsenic, antimony or the phosphorus have five externe electrons. If such atoms are introduced in the crystal lattice of silicon, four valence electrons are used for the binding of the atom foreign with silicon atoms neighbors while the fifth electron is engaged in no bonding 19,20 .

This fifth electron is very little related to arsenic and under the effect of the thermal agitation, normal temperature, it circulates freely as electron conduction within the crystal ${ }^{21}$. The pentavalent atom becomes a positive ion frozen in the crystal. This atom bears the name of the donor because it has increased the number of free electrons. The silicon doped by the donors and as the concentration of free electrons has increased, there is the talk of a doping n: Silicon becomes of ntype silicon. Has the ambient temperature, virtually all donors are ionized and if the concentration in donor atoms is $N_{d}$, the density of the semi-conductor free carriers will by:

$n_{e}=n_{e_{0}}+N_{d}$

\subsection{5. p Doped semiconductor}

If some silicon atoms are replaced by trivalent elements such as boron, indium or gallium, of the connections of the impurity with the silicon atoms neighbors cannot be adequately achieved because it lacks a valence electron. The hole thus created moves by thermal agitation ${ }^{22}$. The impurities having then their four saturated connections have become negative ions. These impurities frozen in the crystal are called acceptors because they tend to capture (accept) the fourth electron. The silicon-doped by the acceptors is called of p-type Silicon because the number of holes has increased 21,23 . The holes are therefore the majority carriers in the silicon of p-type. In the same way as for the semiconductor doped n, the concentration in holes of the semi-driver will by ${ }^{24}$ :

$n_{h}=n_{h_{0}}+N_{a}$

\subsubsection{Semiconductor compensated}

The doping impurities (or even deep) of different type can offset, partially or totally. The semiconductor will be the type of the dominant impurity. If it comes to 
compensate correctly $\left(N_{a}=N_{d}\right)$, then it is an intrinsic semi-conductor by compensation (although it contains impurities doping agents $)^{13}$.

\section{Organic Photovoltaism}

Organic solar cells are developing rapidly, and their conversion efficiencies have recently surpassed the $8 \%$ mark (for comparison, amorphous silicon photovoltaic achieves efficiencies of $12 \%$ in the laboratory).

The most widely used materials in the field of organic photovoltaics polymer-based mixture are poly(3hexylthiophene) (P3HT):[6,6]-phenyl-C61-butyric acid methyl ester (PCBM). The active layer is heterojunction on volume of interpenetrating domains electron acceptor (PCBM) and electron donor $(\mathrm{P} 3 \mathrm{HT})^{14}$.

The photoelectric properties of matter were and are still the subject of increased attention by their

applications in the fields of photoelectricity and conversion of light energy into electrical energy in conventional inorganic semiconductors which unfortunately reached their limits due to technological difficulties manifest their jobs. That is why it was proposed to use organic molecular materials that have some exploitable electronic conductivity in batteries and photovoltaic cells and can be sources of clean, inexhaustible renewable energy if we can master the technology design and manufacturing and to overcome the technical difficulties that may arise ${ }^{25}$.

It is in this context that we will develop the theoretical concepts and the technical procedures that allow the development of organic photovoltaic cells based on photosensitive layers of organic polymers (Donor and electron acceptor) ${ }^{18}$.

\section{1. organic photovoltaic effect}

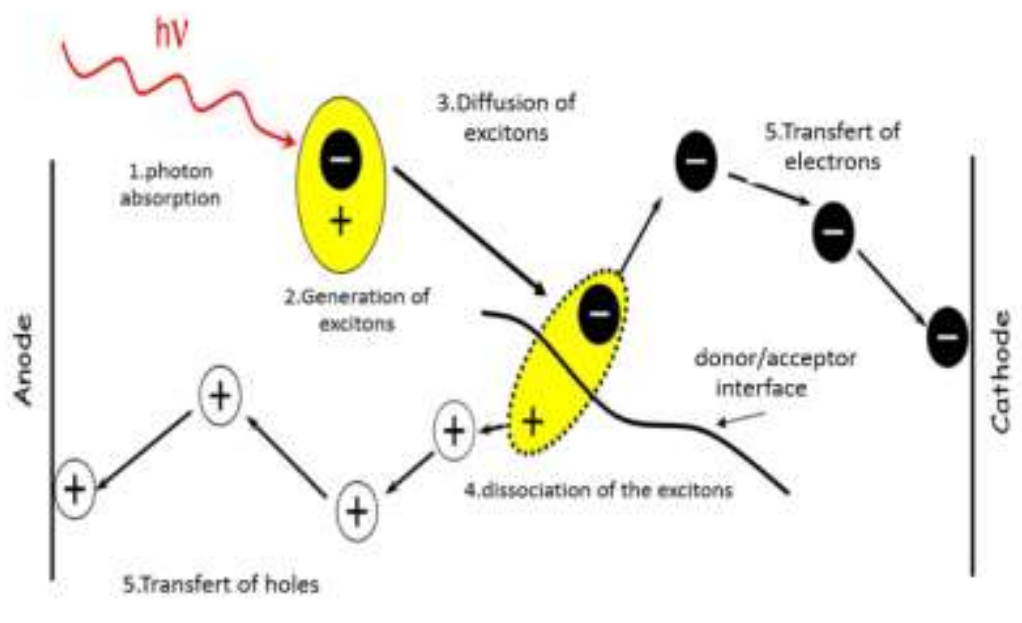

Figure 3. The five stages of photovoltaic conversion in the organic semiconductor.

The key steps governing electronic transfer can be written by the following equations ${ }^{26}$.

Transition training exciton

$\boldsymbol{\pi} \rightarrow \boldsymbol{\pi}^{*}: \boldsymbol{D}+\boldsymbol{A}+\boldsymbol{h} \boldsymbol{v} \rightarrow \boldsymbol{D}^{*}+\boldsymbol{A}$

Relocation of the couple Giver Acceptor :

$\boldsymbol{D}^{*}+\boldsymbol{A} \rightarrow(\boldsymbol{D}-\boldsymbol{A})^{*}$

charge transfer initialization :

$(\boldsymbol{D}-\boldsymbol{A})^{*} \rightarrow\left(\boldsymbol{D}^{\boldsymbol{\delta}+}+\boldsymbol{A}^{\boldsymbol{\delta}-}\right)^{*}$

Forming a pair of radicals :

$\left(D^{\delta+}+A^{\delta-}\right)^{*} \rightarrow\left(D^{+\cdot}+A^{-\cdot}\right)^{*}$

Charge separation :

$\left(D^{+\cdot}-A^{-\cdot}\right)^{*} \rightarrow D^{+\cdot}+A^{-}$

The organic photovoltaic effect is the conversion of light energy into potential difference using cells in organic materials. This effect manifests itself in organic semiconductors, and it can be explained by using the corpuscular aspect of light (assuming that the light is composed of both electromagnetic waves and photons, wave-particle duality).

\subsubsection{Absorption of the photon and the exciton formation}

When photon absorbed by the organic semiconductor, they give rise to a photoionization process and causing an increase of the electron energy and without holes if those are spatially separated, these electron-hole pairs are called excitons which represent a bound state of an electron and hole and which may also be represented by bosons load and zero spin ${ }^{27}$. These excitons diffuse to the donor-acceptor interface of the semiconductor which is essential for electron transfer, because this interface represents the dissociation site of the exciton under the influence of the internal electric field (Figure 3). These excitons must have a long enough life so that they arrive at the interface. 
Indeed, in the organic semiconductor $\left(E_{B} \sim 1 \mathrm{eV}\right.$ for $\left.r_{E} \sim 1 \mathrm{~nm}\right)$.

Considering the exciton as a condition related to an electron and hole, we can deduce its energy levels by $\left[-\frac{\hbar^{2}}{2 m_{e}^{*}} \Delta_{e}-\frac{\hbar^{2}}{2 m_{h}^{*}} \Delta_{h}+\widehat{V}(\right.$ é, $\left.\boldsymbol{h})\right] \boldsymbol{\phi}_{n}($ é $) \phi_{n}(\boldsymbol{h})=\boldsymbol{E}_{n} \boldsymbol{\phi}_{n}($ é $) \phi_{n}(\boldsymbol{h})$

solving the Schrödinger equation on the exciton, which is expressed as follows if:
Since electrons are much more mobile than the holes we can neglect the term of the hole kinetic energy in the Hamiltonian of the exciton and consider him still, which brings us to describe the exciton as a movement of electron in the Coulombian fields of the fixed hole, that is to say replacing the active masses $m_{e}^{*}$ and $m_{h}^{*}$ by the reduced effective mass $\mu^{*}$ which expressed as:

$\mu^{*}=\frac{m_{e}^{*} m_{h}^{*}}{m_{e}^{*}+m_{h}^{*}}$

$-\frac{\hbar^{2}}{2 \mu^{*}} \Delta \psi_{\mathrm{n}}(\mathrm{e}, h)+\widehat{V}(\mathrm{e}, h) \psi_{n}(\mathrm{e}, h)=\boldsymbol{E}_{\boldsymbol{n}}(\mathrm{e}, \boldsymbol{h}) \boldsymbol{\psi}_{\boldsymbol{n}}(\mathrm{e}, \boldsymbol{h})$

Considering the electron-hole interaction as purely Coulombian, one expresses the potential energy operator as if follows:

$\widehat{V}(\mathrm{e}, \boldsymbol{h})=-\frac{e^{2}}{4 \pi \varepsilon r^{2}(\mathrm{e}, h)} \hat{\boldsymbol{r}}$

Moreover, it leads to energy $E_{n}(\mathrm{e}, h)$ levels that are solutions of the Schrödinger equation and have expressed:

$\mathbf{E}_{n}(\mathrm{e}, \boldsymbol{h})=\frac{-\mu^{*} e^{4}}{32 \pi^{2} \hbar^{2} \varepsilon^{2} n^{2}}$

Or $\mu^{*}$ : The effective mass of the exciton; e: the elementary charge; $\varepsilon$ : dielectric constant of the material; $n$ : principal quantum number.

For the radius of the exciton are:

$\boldsymbol{r}_{E}=\frac{4 \pi \hbar^{2} \varepsilon}{\mu^{*} e^{2}} n^{2}$

Also, the binding energy of the exciton is:

$E_{B}=-E_{1}$

It is then findd that the radius of the exciton increases with the dielectric constant to obtain more stable excitons must opt for low dielectric constant materials $(2<\varepsilon<5)$.

The formation of the exciton obeys the law of mass action and can be likened to chemical reaction and can be treated with a thermochemical model:

$\boldsymbol{E}^{*} \rightleftharpoons$ é $+\boldsymbol{h}$

Introduce exciton formation constant:

$\boldsymbol{K}_{\boldsymbol{f}}=\frac{\boldsymbol{n}_{\mathrm{e}} \boldsymbol{n}_{\boldsymbol{h}}}{\boldsymbol{n}_{\boldsymbol{E}^{*}}} \boldsymbol{n}_{\mathrm{e}} \boldsymbol{n}_{\boldsymbol{h}}$

Depending on which to express its free energy of formation:

$\Delta G=2 \pi \hbar N_{A} v \ln \left(K_{f}\right)$

where is the frequency of the incident photons $v$, and $\boldsymbol{N}_{\boldsymbol{A}}$ is Avogadro number.
Then replace the wave functions $\phi_{n}($ é $)$ and $\phi_{n}(h)$ describing the electron and hole wave function $\psi_{\mathrm{n}}(\mathrm{e}, h)$ describing the state of the exciton which is a linear combination of the two functions $\phi_{n}(\mathrm{e})$ and $\phi_{n}(\mathrm{~h})$ :

$\psi_{\mathrm{n}}\left(\mathrm{é}^{\prime}, \boldsymbol{h}\right)=\frac{1}{\sqrt{2}}\left(\phi_{n}(\mathrm{e})+\phi_{n}(\mathrm{~h})\right)$

The Schrödinger equation takes the following form

Consider the formation energy as equal to the electrostatic interaction energy electron-hole sign changed:

$\Delta G=\left|-\frac{e^{2} N_{A}}{4 \pi \varepsilon r_{(\mathrm{e}, h)}}\right|$

This leads to the expression of the constant exciton formation depending on the wavelength of the incident photons and as a function of the exciton radius:

$\ln \left(K_{f}\right)=\frac{e^{2}}{8 \pi^{2} \hbar c \varepsilon} \frac{\lambda}{r_{(e ́, h)}}$

By numerically expressing the constants contained in the above formula is obtained in the formula below according to which the natural logarithm of $\boldsymbol{K}_{\boldsymbol{f}}$ varies linearly with the wavelength as well as the inverse of the exciton radius:

$\ln \left(K_{f}\right)=7,2072.10^{6} \frac{\lambda}{r_{(e ́, h)}}$

Exciton formation can also be schematized in a kinetic model, the recombination rate of excitons in this model expresses the rate of excitons formation:

$R_{\text {éh }}=-\frac{\mathrm{dn}(\mathrm{e} ; \mathrm{h})}{d t}=\frac{n(\mathrm{e} ; \boldsymbol{h})}{\tau}$

As $\tau$ is the lifetime of the exciton defined by:

$\boldsymbol{\tau}=\frac{\Gamma}{\hbar}$

Or $\Gamma$ is the width of the excitonic energy levels.

For biomolecular recombination $R_{\text {éh }}$ can be expressed in terms of the thermodynamic formation constant $K_{f}$ :

$R_{\text {éh }}=\frac{q^{2}}{k T \varepsilon}\left(D_{e}+D_{h}\right) K_{f}$

Can be due to quantum mechanics determine the probability of forming the exciton according to the electric field of the wave associated with the incident photon under formula Keldysh : 
$P_{f}=1-\frac{\lambda E_{B}}{\pi \hbar c}\left[\left(1+\frac{q^{2} E_{0}^{2} \lambda^{2}}{4 \pi^{2} \mu^{*} E_{B} c^{2}}\right) \operatorname{Arsh}\left(\frac{\sqrt{8 \pi^{2} \mu^{*} E_{B}}}{q E_{0}} \frac{c}{\lambda}\right)-\frac{q E_{0} \lambda}{c} \sqrt{\frac{1+\frac{8 \pi^{2} \mu^{*} E_{B} c^{2}}{q^{2} E_{0}^{2} \lambda^{2}}}{32 \pi^{2} \mu^{*} E_{B}}}\right]$

$E_{0}$ : The amplitude of the electric field of the wave associated with the photon.

$\mathrm{C}$ : velocity of light in vacuum.

\subsubsection{Diffusion and dissociation of the exciton}

Following its formation, Pervasive exciton to the donor-acceptor interface or it dissociates into electrons and holes under the action of the electric field prevailing at the D/A interface, the electrons diffuse to the PCBM acceptor and the holes toward the P3HT donor following the laws of diffusion and migration under the influence of the electrochemical potential gradient :

$$
\boldsymbol{\eta}=\boldsymbol{\mu}+\boldsymbol{e \phi}
$$

This separation takes place at the donor-acceptor interface under the influence of the electric field of the gradient of concentration of charge carriers which appears subsequently to the illumination of the donor material whose electrical neutrality is no longer met the diffusion currents result in the creation of the internal electric field, which expressed as:

$\vec{E}=-\frac{K T}{q}\left(\frac{\mu_{e} \frac{d n_{e}}{d x}-\mu_{h} \frac{d n_{h}}{d x}}{n_{e} \mu_{e}+n_{h} \mu_{h}}\right)$

From the laws of quantum mechanics can assimilate the influence of this field prevailing donor-acceptor interface excitons to a perturbation that lifts the degeneracy of their energy levels and that distorts the exciton thereby generating an induced dipole moment which is derived from the theory of perturbation and whose expression for the ground state:

$<\vec{p}>=\frac{9}{2}\left(\frac{4 \pi \hbar^{2} \varepsilon}{\mu^{*} e^{2}}\right)^{3} \vec{E}$

This polarization of the exciton finally leads to its dissociation with a probability dependent on the intensity of this field has been calculated using perturbation theory:

$P_{d}=\frac{q E}{\sqrt{8 \mu^{*} E_{B}}} e^{-\left(\frac{\mu^{* 1 / 2}\left(2 E_{B}\right)^{3 / 2}}{3 q \hbar E}\right)}$

\subsubsection{Generating electrons and holes and their diffusion through the donor-acceptor interface}

After the dissociation of the excitons into electrons and holes under the influence of electrochemical gradient, there is a drift and diffusion of electrons $(D \rightarrow A)$. For the holes, the same phenomena occur but in the opposite direction $(A \rightarrow D)$.

To achieve the Nernst-Planck equations related to the total current of electrons and holes due to the gradient of the electrochemical potential and taking into account the existence of displacement current, the electrochemical potential takes the form:

$\eta=\mu+q \phi+\frac{\varepsilon}{\mu n} \frac{\partial \phi}{\partial t}$

firstly calculation of this gradient:

$\frac{\partial \eta}{\partial x}=\frac{\partial \mu}{\partial x}+q \frac{\partial \phi}{\partial x}-\frac{\varepsilon}{\mu n} \frac{\partial E}{\partial t}$

Substituting the chemical potential $\mu$ by its expression $\mu=\mu_{0}+\frac{k T}{n} \int \frac{d n}{n}$

$\frac{\partial \eta}{\partial x}=\frac{k T}{n} \frac{\partial n}{\partial x}+q \frac{\partial \phi}{\partial x}-\frac{\varepsilon}{\mu n} \frac{\partial E}{\partial t}$

Also, introducing the electro diffusion Rayleigh force

$\mathcal{F}_{R}=-\frac{\partial \eta}{\partial x}$

The current density expressed in terms of the Rayleigh force like the following:

$J=\mu n \mathcal{F}_{R}=-\mu n \frac{\partial \eta}{\partial x}$

$J=-\left[k T \mu \frac{\partial \eta}{\partial x}+q \mu n \frac{\partial \phi}{\partial x}+\varepsilon \frac{\partial E}{\partial t}\right]$

Under the Nernst-Einstein relation

$\frac{D}{\mu}=\frac{k T}{q}$

it was for $k T \mu=D q$ and expression of the total current of electrons takes the form :

$J_{e}=q D_{e} \frac{\partial n_{e}}{\partial x}-q \mu_{e} n_{e} \frac{\partial \phi}{\partial x}+\varepsilon \frac{\partial E}{\partial t}$

For the holes by the same calculation method are:

$J_{h}=-q D_{h} \frac{\partial n_{h}}{\partial x}-q \mu_{h} n_{h} \frac{\partial \phi}{\partial x}+\varepsilon \frac{\partial E}{\partial t}$

The first term of the two equations is the diffusion current; the second term is the current drift, and the third term is the displacement current.

The total current is the sum of two currents related to electrons and holes:

$J=q D_{e} \frac{\partial n_{e}}{\partial x}-q \mu_{e} n_{e} \frac{\partial \phi}{\partial x}-q D_{h} \frac{\partial n_{h}}{\partial x}-q \mu_{h} n_{h} \frac{\partial \phi}{\partial x}+2 \varepsilon \frac{\partial E}{\partial t}$

numbers; by calculation of the divergence of the current density:

$\operatorname{div} J=q D_{e} \frac{\partial^{2} n_{e}}{\partial x^{2}}+q \frac{\partial D_{e}}{\partial x} \frac{\partial n}{\partial x}-q \mu_{e} n_{e} \frac{\partial^{2} \phi}{\partial x^{2}}-q \mu_{e} \frac{\partial n}{\partial x} \frac{\partial \phi}{\partial x}+\varepsilon \frac{\partial d i v E}{\partial t}$

According to Maxwell-Gauss equation

$\operatorname{div} E=\frac{\rho}{\varepsilon}=\frac{q n}{\varepsilon}$ 
We can write the div J depending on the evolution of

the density concerning the time.

$\operatorname{div} J=q D_{e} \frac{\partial^{2} n_{e}}{\partial x^{2}}+q \frac{\partial D_{e}}{\partial x} \frac{\partial n}{\partial x}-q \mu_{e} n_{e} \frac{\partial^{2} \phi}{\partial x^{2}}-q \mu_{e} \frac{\partial n}{\partial x} \frac{\partial \phi}{\partial x}+q \frac{\partial n}{\partial t}$

Using the fourth Maxwell equation)

$\operatorname{rot} B=\mu_{0}\left(q D_{e} \frac{\partial n_{e}}{\partial x}-q \mu_{e} n_{e} \frac{\partial \phi}{\partial x}+\varepsilon \frac{\partial E}{\partial t}\right.$

Also, knowing that $\operatorname{divrot} B=0 \quad$ then $\operatorname{divJ}=0$ Assuming that $D_{e}$ is independent of the thickness, the second term of the equation vanishes, and can then write :

$D_{e} \frac{\partial^{2} n_{e}}{\partial x^{2}}-\mu_{e} n_{e} \frac{\partial^{2} \phi}{\partial x^{2}}-\mu_{e} \frac{\partial n}{\partial x} \frac{\partial \phi}{\partial x}+\frac{\partial n}{\partial t}=0$

After the illumination we must include the recombination of electrons and holes in the excitons at the Donor (P3HT) and their generation later dissociation of these to the donor-acceptor interface, the balance of conservation the number of charge carriers then written to the electrons:

$D_{e} \frac{\partial^{2} n_{e}}{\partial x^{2}}-\mu_{e} n_{e} \frac{\partial^{2} \phi}{\partial x^{2}}-\mu_{e} \frac{\partial n_{e}}{\partial x} \frac{\partial \phi}{\partial x}+\frac{\partial n_{e}}{\partial t}+G_{e}-R_{e}=0$

In steady-state the continuity equation becomes:

$D_{e} \frac{\partial^{2} n_{e}}{\partial x^{2}}-\mu_{e} n_{e} \frac{\partial^{2} \phi}{\partial x^{2}}-\mu_{e} \frac{\partial n_{e}}{\partial x} \frac{\partial \phi}{\partial x}+G_{e}-\frac{n_{e}}{\tau}=0$

The same calculations can be made to derive the continuity equation for holes:

$D_{e} \frac{\partial^{2} n_{e}}{\partial x^{2}}-D_{h} \frac{\partial^{2} n_{h}}{\partial x^{2}}-\mu_{e} n_{e} \frac{\partial^{2} \phi}{\partial x^{2}}-\mu_{h} n_{h} \frac{\partial^{2} \phi}{\partial x^{2}}-\mu_{e} \frac{\partial n_{e}}{\partial x} \frac{\partial \phi}{\partial x}-\mu_{h} \frac{\partial n_{h}}{\partial x} \frac{\partial \phi}{\partial x}+G_{e}+G_{h}-\frac{n_{e}}{\tau_{e}}-\frac{n_{h}}{\tau_{h}}=0$

By this reasoning is yielded to the universal equations governing the charge carrier movement under the influence of the gradient of the electrochemical potential and the formation and dissociation of excitons which are essential to charge transfer from the donor to the acceptor.

Regarding the electric field, its expression can be deduced from the expressions of electric currents diffusion and charge carrier migration deducted above:

$J_{e}=q n_{e} \mu_{e} E+\mu_{e} k T \frac{d n_{e}}{d x}$

$J_{h}=q n_{h} \mu_{h} E-\mu_{h} k T \frac{d n_{h}}{d x}$

If open circuit the sum of the two currents is zero and results in the expression of the electric field:

$J_{e}+J_{h}=\mathbf{0}$

If the semiconductor is monopolar $\left(n_{h}=0\right)$ expression of the electric field reduced to:

$E=-\frac{k T}{q} \frac{1}{n_{e}} \frac{d n_{e}}{d x}$

Integrating $E$ over $\mathrm{dx}$ found expression broadcast Nernst potential:

$\phi=\frac{k T}{q} \ln \left(\frac{n_{e 2}}{n_{e 1}}\right)$
$\boldsymbol{n}=\boldsymbol{n}_{e}+\boldsymbol{n}_{G e}-\boldsymbol{n}_{\boldsymbol{R} e}$

The evolution equation of the density in time:

$\frac{\partial n}{\partial t}=\frac{\partial n_{e}}{\partial t}+\frac{\partial n_{G e}}{\partial t}-\frac{\partial n_{R e}}{\partial t}=\frac{\partial n_{e}}{\partial t}+G_{e}-R_{e}$

where $R_{e}$ also, is the rate of recombination

$\boldsymbol{G}_{\boldsymbol{e}}$ is the generation rate of electrons:

$\boldsymbol{G}_{\boldsymbol{e}}=\alpha(\boldsymbol{x}) \boldsymbol{\phi}(\lambda)(1-R(\lambda)) \boldsymbol{e}^{-\alpha(\lambda) x}$

where $\boldsymbol{\alpha}(\boldsymbol{x})$ the absorption coefficient.

$\phi(\lambda)$ : the incident flux.

$\boldsymbol{x}$ : the position.

$\boldsymbol{R}(\lambda)$ : the reflectivity.

Finally, the continuity equation takes the form

$-D_{h} \frac{\partial^{2} n_{h}}{\partial x^{2}}-\mu_{h} n_{h} \frac{\partial^{2} \phi}{\partial x^{2}}-\mu_{h} \frac{\partial n_{h}}{\partial x} \frac{\partial \phi}{\partial x}+G_{h}-\frac{n_{h}}{\tau} 0$

The continuity equation for the conservation of the total number of charge carriers in the form:

\subsubsection{Electron transfer and charge separation}

Let us now transfer the electron donor to the acceptor which can take place either by exchange or by resonance through delocalized bonds $\pi$, or by tunnel effect ${ }^{17}$.

Conduction necessary for a charge transfer is possible is that the difference between the donor ionization energies and electronic affinity of the acceptor must be lower or equal to the end of their Coulomb interaction.

The donor exchange transfer probability to the acceptor if the interaction assimilates D / A to form a charge transfer complex is given by:

$P_{D \rightarrow A}=\frac{\left|\left\langle\psi_{D}|\widehat{\beta}| \psi_{A}\right\rangle\right|^{2}}{\left(E_{D}-E_{A}\right)^{2}}$

$\psi_{D}$ and $\psi_{A}$ are the donor wave functions respectively and acceptor

$\hat{\beta}$ : The exchange integral D / A

$E_{D}$ et $E_{A}$ are respectively the energy of the donor and the acceptor before the electronic transfer (isolated). Determining the energy of the HOMO-LUMO transition by the Huckel method by introducing the bonding $\pi$ orbital. 
$\psi_{\pi}=C_{1}\left(2 P_{\mathrm{z}_{1}}\right)+C_{2}\left(2 P_{\mathrm{z}_{2}}\right)$ as $C_{1}=C_{2}=\frac{1}{\sqrt{2(1+S)}}$

The anti-bonding orbital $\pi *$ :

$\psi_{\left(\pi^{*}\right)}=C_{1}\left(2 P_{\mathrm{z}_{1}}\right)-C_{2}\left(2 P_{\mathrm{z}_{2}}\right)$ as $C_{1}=C_{2}=\frac{1}{\sqrt{2(1-S)}}$

$S$ is the overlap integral of atomic orbitals $2 P_{\mathrm{z}_{1}}$ and $2 P_{\mathrm{z}_{2}}$ with:

$S=\left\langle 2 P_{\mathrm{z}_{1}} \mid 2 P_{\mathrm{z}_{2}}\right\rangle$

Calculating the diagonal matrix elements of the Hamiltonian $\widehat{H}$ using $\psi_{\pi}$ and $\psi_{\left(\pi^{*}\right)}$ by Huckel method to reach the energy $E_{\pi}$ and $E_{\pi^{*}}$ before the transition:

$$
\begin{aligned}
& E_{\pi}=\left\langle\psi_{\pi}|\widehat{H}| \psi_{\pi}\right\rangle=\alpha+\beta \\
& E_{\pi^{*}}=\left\langle\psi_{\pi^{*}}|\widehat{H}| \psi_{\pi^{*}}\right\rangle=\alpha-\beta
\end{aligned}
$$

Or $\alpha$ is the integral of Coulomb.

The transition energy HOMO- LUMO acts as the perturbation of the first order of orbital energies $\pi$ :

$$
\left\langle\psi_{D}|\widehat{H}| \psi_{A}\right\rangle=\frac{\hbar^{2} k_{\mathrm{e}}^{2}}{2 m_{\mathrm{e}}^{*}}+\frac{\hbar^{2} k_{h}^{2}}{2 m_{h}^{*}}+\boldsymbol{V}(\mathbf{e}, \boldsymbol{h})+V_{c}+\mathbf{E}_{\mathbf{v i b}_{\mathrm{r}}}
$$

$k_{\mathrm{e}}$ and $k_{\mathrm{h}}$ are the wave vectors of the electrons and holes $V(e ́, h)$ The electron-hole interaction

$$
\mathbf{E}=\frac{\mid\left\langle\boldsymbol{\psi}_{\pi}|\widehat{\boldsymbol{H}}| \boldsymbol{\psi}_{\pi *}||^{2}\right.}{E_{\pi}-E_{\pi^{*}}}
$$

The become the energy of the absorbed photon:

$\mathbf{2 \pi} \hbar \boldsymbol{v}=\mathrm{I}(\mathrm{D})-\mathbf{E}(\mathrm{A})+\left\langle\boldsymbol{\psi}_{D}|\widehat{H}| \boldsymbol{\psi}_{A}\right\rangle$

Or I(D) is the donor ionization energy:

$\mathrm{I}(\mathrm{D})=\boldsymbol{\alpha}-\boldsymbol{\beta}$

$\mathrm{E}(\mathrm{A})$ Is the electron affinity of the acceptor

The third term, therefore, includes a term Coulomb

$V_{c}=-\frac{q_{D} q_{A}}{4 \pi \varepsilon \delta_{D A}}$

The first two terms are the kinetic energies of the electrons and holes:

$\mathrm{E}_{\mathrm{vib}_{\mathrm{r}}}$ The energy transmitted to the vibrational levels of the polymer chains.

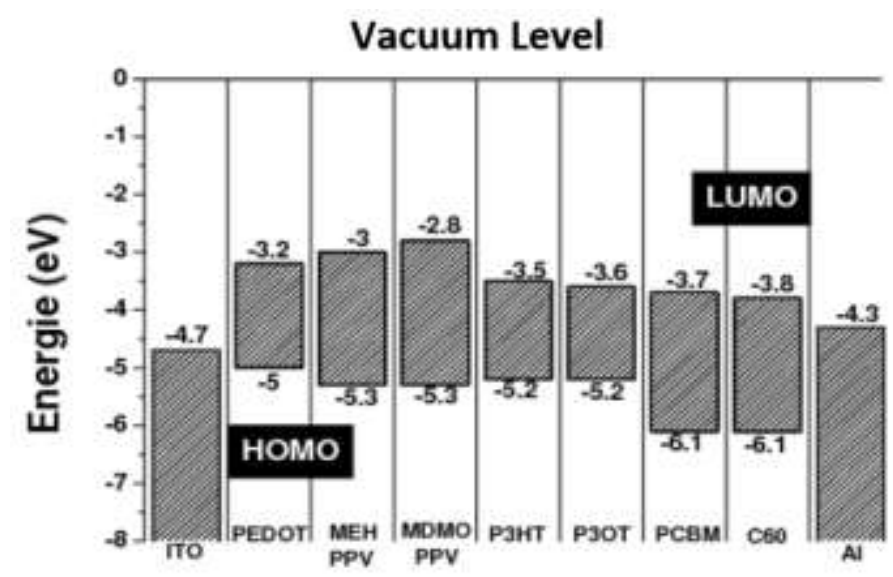

Figure 4. Diagrams of energy bands of conjugated polymers and acceptors used in photovoltaic cells.

The electron then undergoes a transition from the valence band to the conduction band, the energy needed for this transition is the energy gain due to the establishment of a bond between the donor site of
P3HT and the acceptor site of PCBM (Figure 4).

This energy is given by equation Klopman, after the frontier orbital theory:

$\Delta E=-\frac{\left|q_{A} q_{D}\right|}{4 \pi \varepsilon \delta_{D A}}+2 \sum_{H о м о(D)} 2 \sum_{H о м о(D)} \frac{\left|c_{D}^{H O M O} C_{A}^{L U M O} \Delta \beta_{D A}\right|^{2}}{E_{D}-E_{A}}$

The transfer of electrons is governed by the electrostatic interaction of the opposite charges and if $E_{D}-E_{A}$ large, and by the interaction between the frontier orbitals is $E_{D}-E_{A}$ so small, and it is this electron flow which is the photovoltaic current in an organic semiconductor. But the question is how this electronic transfer is possible? Also, why are these electrons so labile?
Before answering these questions we must first mention that there are two types of organic semiconductors ${ }^{28}$ : The molecular semiconductors and macromolecular semiconductors (Figure 5), photoconductivity and semiconductivity in these semiconductors classes are due to the conjugation present in these compounds phenomenon which tends to delocalize the electrons along the polyene chains 
and polymer chains scheme below illustrates some examples of receptor molecules and electron-donor molecules and electron as sees all these molecules are

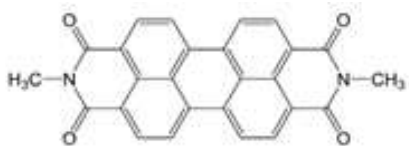

MePTCDI

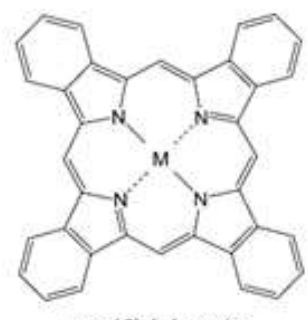

metal Phthalocyanine

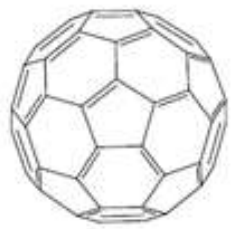

C60 conjugated, and have labile $\pi$ electrons which absorb in the visible and ultraviolet and which impart the property of the semiconductivity to these compounds.

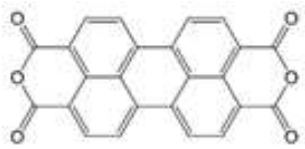

PTCDA

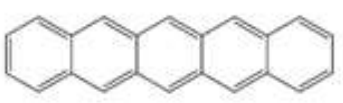

Pentacene

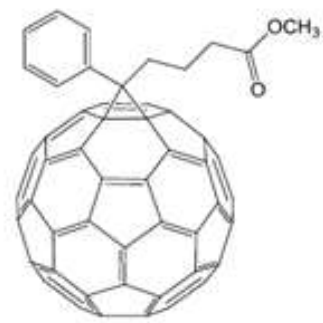

PCBM

Figure 5. Examples of small conjugated molecules commonly used in organic photovoltaics.

The compound of choice studied the photoactive layer are P3HT: poly (3-hexylthiophene) and PCBM: [6,6] phenyl-C61-butyric acid methyl ester.

To amplify the combination of effects, it makes covalent grafting between PCBM and $\mathrm{C}_{60}$ fullerene ${ }^{29}$.

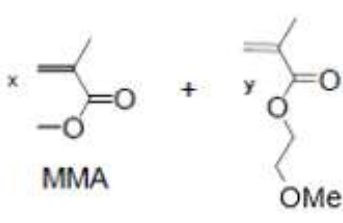

HEMM

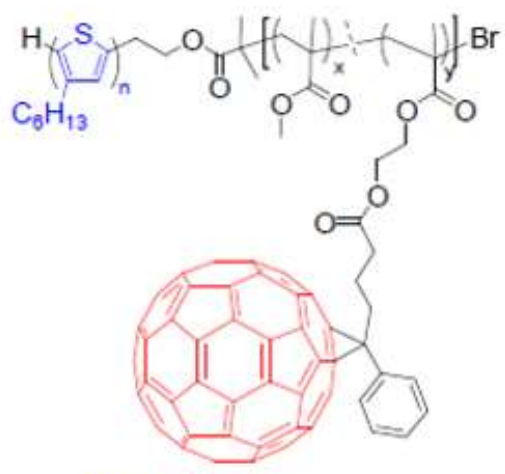

P3HT - $\mathrm{PCBM}$

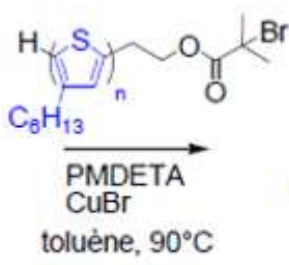

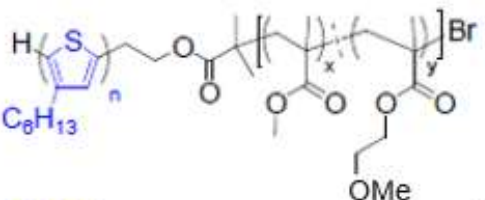

P3HT

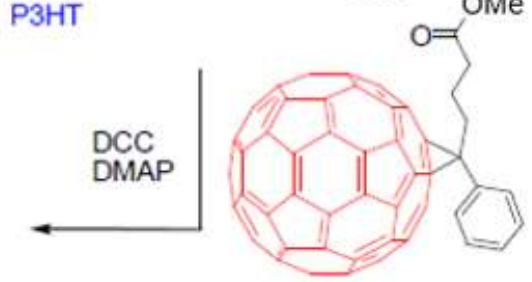

PCBM

Figure 6. esterification of $\mathrm{P} 3 \mathrm{HT}$ in the presence of PCBM.

In this allotropic form of carbon it alternately enters hexagonal rings of carbon atoms and pentagonal rings making long delocalize the electrons of the C60-MBP molecule. So the presence of $\pi$ conjugation and orbital 
phenomena are crucial to the lability of electrons transferred from the donor P3HT to the acceptor PC60BM (Figure 6).

Electronic terms of the active layer molecules interact with the vibrations of the molecular chain (orbit coupling -Vibration). This results in the training of electrons by phonons (quasiparticle boson quantifying the vibrations of a material medium) of other hands the existing internal electric field in the donoracceptor interface and the diffusion layer. Furthermore, the electric field created by the charges transferred leads to the deformation of the electron cloud of the molecule. So the polarization of the latter and creating traps potential well for electrons, which results in the formation of polarons (polaron: + electron potential well),that can circulate in the molecule and which can form pairs positive polaronnegative polaron (bipolaron) that tend to locate the charges on some polymer chains and therefore facilitate the transfer of electrons. The diagram below illustrates the sites of absorption photons (the $\pi$ electrons) and formation of excitons which are relocating along the polymer chain as well as trapping sites of electrons $(\sigma-\pi-\sigma)$ and the formation of polarons $^{23}$ (Figure 7).

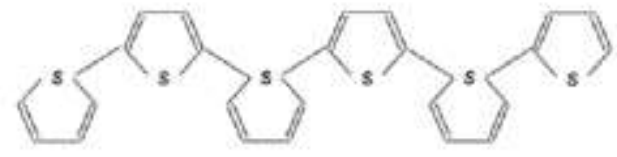

Polythiophene

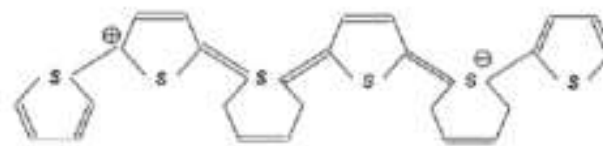

Exciton

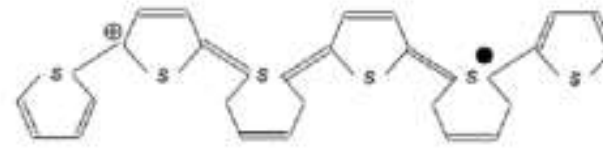

Positif Polaron

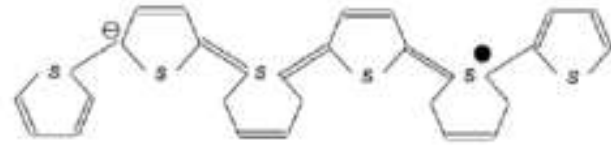

Negatif Polaron

Figure 7. Formation of a positive and negative polaron in a polythiophene

If we can reduce the thickness of the Donor-Acceptor interface nanoscale $\left(\delta_{D A} \sim \lambda_{\text {DeBroglie }}\right)$, another possibility of electron transfer is possible is the tunnel effect due to the wave aspect of electrons. Also in polyurethanes under well-defined conditions of the medium (the effect of salt, $\mathrm{pH}$, ionic force, solvent polarity, the internal electric field). These molecules may curl and twist in $\alpha$ helix or $\beta$-sheet by the formation of hydrogen bonds between the $\mathrm{CO}$ carbonyl groups and amine groups $\mathrm{NH}$; electrons can thus be transferred by tunnel effect through these hydrogen bonds, so the conformational transitions $\alpha \Leftrightarrow \beta$ further facilitate the transfer of electrons.

\subsection{Organic materials semiconductor applications}

The organic semiconductor materials are the starting point of a new branch of electronics, which is molecular electronics. Their applications are developed and enriched among the most promising applications of the organic semiconductor. Including the conductivity of field-effect transistor of carbon nanotube and the logic gates from fullerene that can be used for medical purposes especially in neutralizing the virus. This involves grafting certain molecules fullerene that can recognize certain viral capsid proteins and therefore complex with the virus and neutralize it ${ }^{19,30}$.

Also, these logic gates may be used to transmit boolienne information in connection with the closing and opening of the molecule cycles after that its complexation with another molecule labeled (radioactivity, fluorescence, paramagnetism ...). Another application in the field of electronics and computer is to use molecular ionic organic semiconductors to develop artificial ion channels similar to those found in biological membranes; these channels may function as ionic microprocessors based on closure and opening ${ }^{31}$.

In cryptography can be used for these channels to encode information in boolienne form, as well as for the design of a digital cellular automaton two discrete dynamic states and which may be considered to model the polymerization reactions, biological morphogenesis and crystal growth ${ }^{32}$.

It is also conceivable very interesting medical applications of the carbon foam is an allotrope of carbon consisting of heptagonal cycles which gives this foam two remarkable properties of semiconductivity (electrons $\pi$ ) paramagnetism (single electron) ${ }^{32}$. Another biological application is the 
magnetography which allows medical diagnosis by recording the magnetic fields created by the biological tissue using a non-destructive biological magnetoresistive sensor based on this foam, as well as imaging contrast factor resonance electron paramagnetic. Another biological application is the magneto-probe microscopy (carbon foam) which can be used as a marker precursor in metabolomics playing an essential role in medicine ${ }^{33}$.

In the field of photo-electrochemical and solid electrochemistry, organic semiconductors can be used as electrodes for the photo-electrosynthesis and organic electrosynthesis (amino acids, amino alcohols, electro-polymerisation...). For the manufacture of photo-electrolyzers water used for energy purposes, electrolysers, galvanic cells with solid electrolyte and miniaturized solar cells that have broad applications in the field of astronautics as energy sources for satellites and space shuttles (NASA Horizon project for the exploration of pluto 2001-2015).

Moreover, these materials may be considered for the implementation of OLEDs (organic light-emitting diode) and organic tunnel diodes, as well as magnetic transistors based carbon foam which serve as transducers in chemical and biological sensors used for analysis and immediate assays (medicine, food, environment ...) ${ }^{21}$.

Fullerenes such can be used thanks to their antioxidant, and anti-free radical in therapeutics for the treatment of brain tumors (gliomas, astrocytomas) made that these molecules can cross the bloodenkephalin brain barrier, as well as unmanned vectors drugs ${ }^{34}$ (absence of side effects due to the dispersion of the drug in the body).
Many of organic semiconductors can be used for developing nanoscale films that can be used for the design of tunnel spectrometers and excitons molecular microscopes based on the focusing end photon radiative dissociation of excitons in a crystal of anthracene. Their trappings in well polaritonic potential, these excitonic light sources also allow visualization of chromatograms and electropherograms across pico-gramme and Femto gramme $^{28}$.

One of the latest applications of organic semiconductivity comes from the low-temperature physics, the bipolaronic superfluid, an exotic state of matter due to a collective quantum phenomenon that is the condensation of Bose-Einstein of bipolarons ${ }^{36}$.

\section{Numerical applications and results}

Numerical calculations were performed and the graphs were obtained by using the Mathematica- 8 software. Numerical results obtained were compared with previous work in the literature, only in this work equations on the excitons was applied by treating them with ion pairs or similar electron-hole bound states at the hydrogen atom by Denison and Ramsay against the work has focused on the ion pairs in solution ${ }^{37}$.

To calculate the exciton dissociation section of it inspired by the work of LD Landau ${ }^{38}$. In calculating the scattering sections (scattering) based on the formula of M.BORN (1926) ${ }^{39}$.

For the calculation of the internal electric field were employed values of electrons and holes mobilities of their densities and the thickness of the D/ A interface.

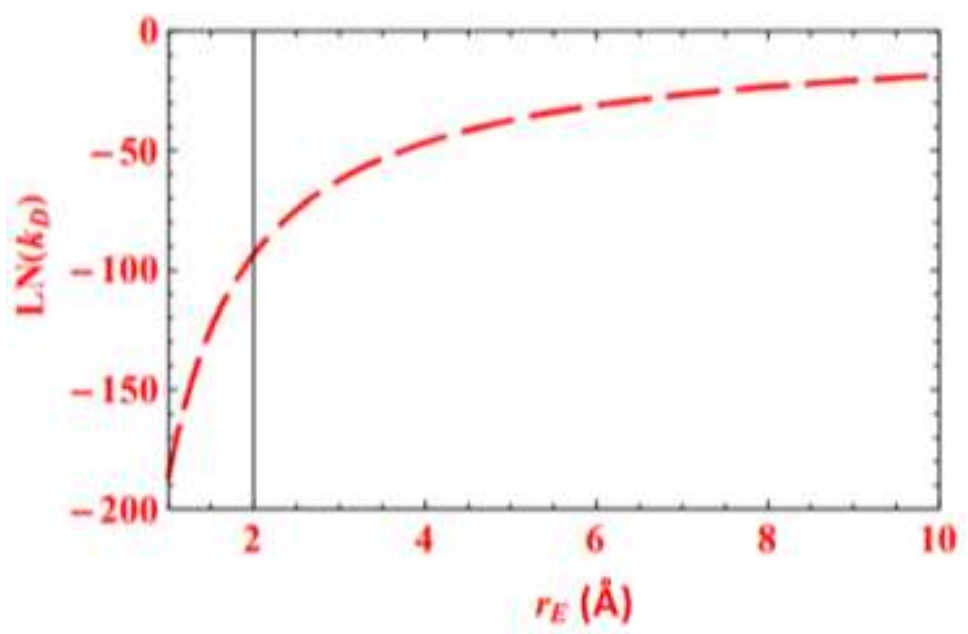

Figure 8. logarithm of the dissociation constant of the exciton according to its radius.

This graph illustrates the variation of the natural logarithm of the dissociation constant (association constant) of the exciton according to its radius, obtained from an electrostatic model assimilating the dissociation of the exciton to electrolytic dissociation (Figure 8). 
These graphs were obtained by drawing on the work of Denison and Ramsay for ion pairs:

$-\ln K_{D}=\frac{e^{2}}{4 \pi \varepsilon k T} \frac{1}{r_{E}}$

According to the graphs the logarithm of the dissociation constant of the exciton $K_{D}$ increases with radius, therefore increase with the radius and this means a decrease in the binding energy of the exciton,

so $K_{D}$ the exciton is voluminous; it is more easily severable that is to say, the stability of the exciton controlled by its size.

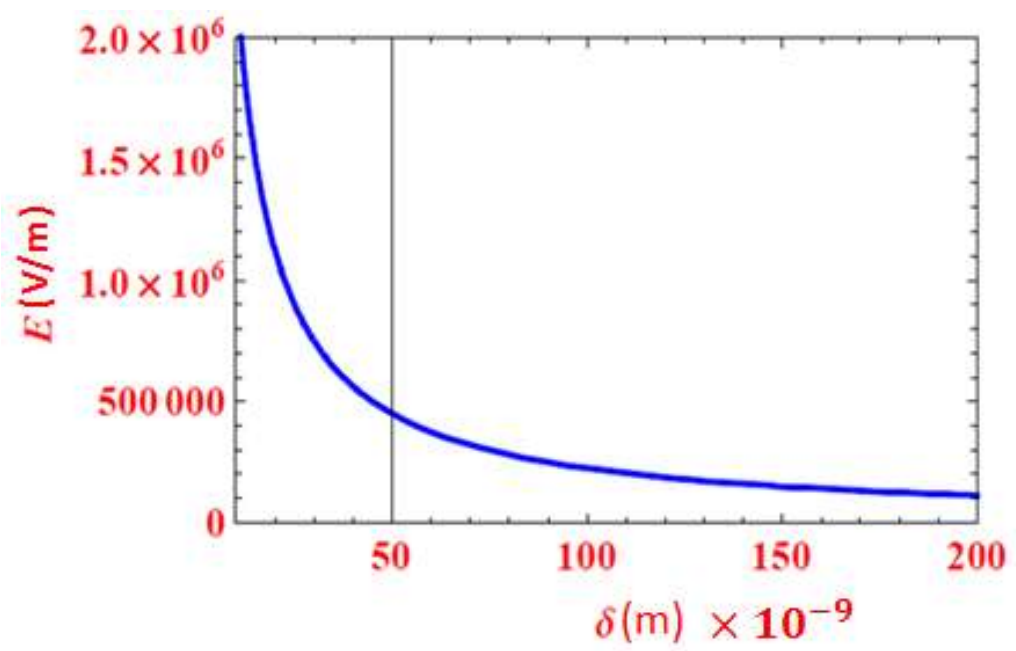

Figure 9. Variation of the internal electric field based on the thickness of the D / A interface

The variation graph of the internal electric field has the appearance of hyperbole and this implies that the internal electric field decreases with increasing thickness of the D / A interface (Figure 9), this decrease is very fast in the meantime $\left(5 \times 10^{5}-2 \times 10^{6}\right)$ then becomes much slower. So we must opt for small thicknesses of the D / A interface to obtain intense electric fields likely to dissociate excitons and facilitate the diffusion and drift of electrons in the D / A interface.

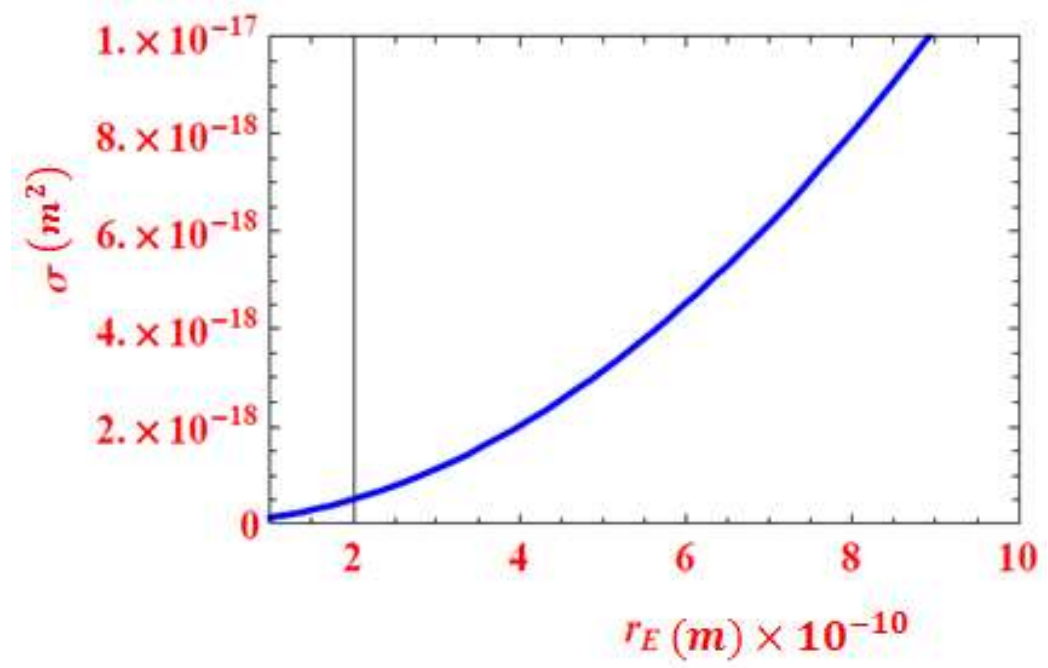

Figure 10. The exciton dissociation section as a function of its radius.

This graph describes the variation of the exciton dissociation section according to its radius (Figure 10), from the graph it is seen that the separation section is a parabolic function with increasing radius than the exciton is more extensive than its dissociation section is large by server for the calculation by the equation deduced from the Born approximation:

$$
\sigma=16 \pi r_{E}^{2} \frac{1}{4\left(\frac{2 \mu^{*} E_{B}}{\hbar^{2}} r_{E}^{2}+1\right)}
$$

It should be noted that each numerical value used for the radius in the calculation of the section is a value of the binding energy $E_{B}$ is another effective mass $\mu^{*}$ due to the dependence of $r_{E}$ and $E_{B}$ 
Table 1. The calculations are based on numerical parameters below.

\begin{tabular}{|c|c|}
\hline Parameters & Values \\
\hline $\begin{array}{l}\text { The exciton effective mass }(\mathrm{Kg}) \boldsymbol{\mu}^{*} \\
\qquad \boldsymbol{F o r} \boldsymbol{r}_{\boldsymbol{E}}=\mathbf{1 0} \AA \text { and } \boldsymbol{E}_{\boldsymbol{B}}=\mathbf{1 e V}\end{array}$ & $0,34716 \cdot 10^{-31}$ \\
\hline $\begin{array}{l}\text { The electron mobility of in the } \\
\operatorname{PCBM} \boldsymbol{\mu}_{\boldsymbol{e}}\left(\boldsymbol{m}^{\mathbf{2}} \cdot \boldsymbol{V}^{-\mathbf{1}} \cdot \boldsymbol{s}^{-\mathbf{1}}\right)\end{array}$ & 3. $10^{-7}$ \\
\hline The hole mobility in the P3HT $\boldsymbol{\mu}_{\boldsymbol{h}}\left(\boldsymbol{m}^{\mathbf{2}} \cdot \boldsymbol{V}^{-\mathbf{1}} \cdot \boldsymbol{s}^{-\mathbf{1}}\right)$ & $2.10^{-8}$ \\
\hline The density of electrons $\boldsymbol{n}_{\boldsymbol{e}}\left(\boldsymbol{m}^{-3}\right)$ & $2.10^{12}$ \\
\hline The density of holes $\boldsymbol{n}_{\boldsymbol{h}}\left(\boldsymbol{m}^{-3}\right)$ & $2.10^{12}$ \\
\hline The interface(D / A) thickness $\boldsymbol{\delta}(\boldsymbol{n m})$ & $10<\delta<200$ \\
\hline dielectric constant $\boldsymbol{\varepsilon}\left(\boldsymbol{F} . \boldsymbol{m}^{-\mathbf{1}}\right)$ & $2,652.10^{-11}$ \\
\hline The reduced Planck constant $\hbar(\boldsymbol{J} . \boldsymbol{s})$ & $1,054 \cdot 10^{-34}$ \\
\hline The Boltzmann constant $\boldsymbol{k}\left(\boldsymbol{J} \cdot \boldsymbol{K}^{-\mathbf{1}}\right)$ & $1,38.10^{-23}$ \\
\hline The temperature $\mathrm{T}(\mathrm{K})$ & 298 \\
\hline The elementary charge q (C) & $1,6.10^{-19}$ \\
\hline
\end{tabular}

\section{General conclusion}

The organic semi-conductivity is a significant challenge for materials science because it is still a vast and rich far from exhausted under his broad yet possible technological applications of energy through molecular electronics and arriving at the complicated applications and more steep in the biomedical field, the nano-electrochemistry and nuclear electrochemistry (nuclear reaction at room temperature).

Moreover, last but not limited to; the design of nanogenerators bioluminescence-photovoltaics (luciferin complexed with a phytochrome) and biomimetics of prebiotic reactions of protein synthesis by adsorption on organic semiconductor films and the effect of light on this process, so a reality that is at the border of reality and who can sometimes significantly exceed fiction.

\section{References}

1- A. Naddaf, S. Zeinali Heris. Experimental study on thermal conductivity and electrical conductivity of diesel oil-based nanofluids of graphene nanoplatelets and carbon nanotubes. Int. Commun. Heat Mass Transf., 2018, 95, 116-122.

2- C.Y. Tso, S.C. Fu, C.Y.H. Chao. A semi-analytical model for the thermal conductivity of nanofluids and determination of the nanolayer thickness. Int. J. Heat Mass Transf., 2014, 70, 202-214.

3- C.J.M. Emmott, D. Moia, P. Sandwell, et al. In-situ, long-term operational stability of organic photovoltaics for off-grid applications in Africa. Sol. Energy Mater. Sol., Cells 2016, 149, 284-293.

4- S.P. Pozdniakov, P. Wang, M.V. Lekhov. A semianalytical generalized Hvorslev formula for estimating riverbed hydraulic conductivity with an open-ended standpipe permeameter. J. Hydrol., 2016, 540, 736-743.
5- A. Tessema, A. Kidane. Cross-property interaction between stiffness, damage and thermal conductivity in particulate nanocomposite. Polym. Test., 2017, 64, 127-135.

6- K. Qi, J.M. Zhao and G.C. Wang, A density functional theory study of ethylene hydrogenation on $\mathrm{MgO}$ - and $\gamma$-Al2O3-supported carbon-containing Ir4 cluster, Physical Chemistry Chemical Physics, 2015,17, 4899-4908.

7- D. Faraco, M. Prats. Characterization for stability in planar conductivities. J. Differ. Equ., 2018, 264 (9), 5659-5712.

8- P.M. Gammon, C.W. Chan, F. Li, et al. Development, characterisation and simulation of wafer bonded Si-on-SiC substrates. Mater. Sci. Semicond. Process., 2018, 78, 69-74.

9- P. Pampili, P.J. Parbrook. Doping of III-nitride materials. Mater. Sci. Semicond. Process., 2017, 62, 180-191.

10- X. Fan, S. Xu, P. Li, et al. Nonpolar and semipolar ultraviolet multiple quantum wells on GaN/sapphire. Mater. Sci. Semicond. Process., 2019, 92, 103-107.

11- K. Qi, S. Liu, M. Qiu,Photocatalytic performance of $\mathrm{TiO} 2$ nanocrystals with/without oxygen defects, Chinese Journal of Catalysis, 2018, 39, 867-875.

12- K. Qi, Q. Qin, X. Duan, G. Wang, L. Wu, W. Zheng, Geometric Matching Principle for Adsorption Selectivity of Ionic Liquids: A Simple Method into the Fascinating World of Shape-Controlled Chemistry, Chemistry - A European Journal, 2014, 20, 29, 9012-9017.

13- K. Zilberberg, F. Gasse, R. Pagui, et al. Highly Robust Indium-Free Transparent Conductive Electrodes Based on Composites of Silver Nanowires and Conductive Metal Oxides. Adv. Funct. Mater., 2014, 24 (12), 1671-1678.

14- C.J.M. Emmott, D. Moia, P. Sandwell, et al. In-situ, long-term operational stability of organic photovoltaics for off-grid applications in Africa. Sol. Energy Mater. Sol. Cells, 2016, 149, 284-293. 
15- K. Qi, J.-M. Zhao, G.-C. Wang. A density functional theory study of ethylene hydrogenation on $\mathrm{MgO}$ - and $\gamma$-Al2O3-supported carbon-containing Ir4 clusters. Phys. Chem. Chem. Phys., 2015, 17 (7), 4899-4908.

16- R. Becker, M. Gebremichael, M. Märker. Impact of soil surface and subsurface properties on soil saturated hydraulic conductivity in the semi-arid Walnut Gulch Experimental Watershed, Arizona, USA. Geoderma, 2018, 322, 112-120.

17- M. Gruenewald, L.K. Schirra, P. Winget, et al. Integer Charge Transfer and Hybridization at an Organic Semiconductor/Conductive Oxide Interface. J. Phys. Chem., C 2015, 119 (9), 4865-4873.

18- S. Jäckle, M. Mattiza, M. Liebhaber, et al. Junction formation and current transport mechanisms in hybrid n-Si/PEDOT:PSS solar cells. Sci. Rep., 2015, 5 (1).

19- S.D. Stranks, H.J. Snaith. Metal-halide perovskites for photovoltaic and light-emitting devices. Nat. Nanotechnol., 2015, 10 (5), 391-402.

20- C.Y. Zhu, Z.Y. Li. Modeling of the apparent solid thermal conductivity of aerogel. Int. J. Heat Mass Transf., 2018, 120, 724-730.

21- S.Z. Oener, J. van de Groep, B. Macco, et al. Metal-Insulator-Semiconductor Nanowire Network Solar Cells. Nano Lett., 2016, 16 (6), 3689-3695.

22- A. Zengin, M. Utku Badak, M. Bilici, Z. Suludere, N. Aktas, Preparation of molecularly imprinted PDMS elastomer for selective detection of folic acid in orange juice, Applied Surface Science, 2019, 471, 162-167.

23- I. Salzmann, G. Heimel, M. Oehzelt, S. Winkler, N. Koch. Molecular Electrical Doping of Organic Semiconductors: Fundamental Mechanisms and Emerging Dopant Design Rules. Acc. Chem. Res., 2016, 49 (3), 370-378.

24- N. Murakami, Y. Kurihara, T. Tsubota, T. Ohno. Shape-Controlled Anatase Titanium(IV) Oxide Particles Prepared by Hydrothermal Treatment of Peroxo Titanic Acid in the Presence of Polyvinyl Alcohol. J. Phys. Chem. C, 2009, 113 (8), 3062-3069.

25- Q. Xu, T. Song, W. Cui, et al. SolutionProcessed Highly Conductive PEDOT:PSS/AgNW/GO Transparent Film for Efficient Organic-Si Hybrid Solar Cells. ACS Appl. Mater. Interfaces, 2015, 7 (5), 3272-3279.

26- H. Kroto, J. Heath, S. Obrien, R. Curl, R.Smalley,C(60) : Buckminister fullerene, Nature, 1985, 318, 162-163.

27- P. Peumans, A. Yakimov and S. R. Forrest, Small molecular weight organic thin-film photodetectors and solar cells, Journal Of Applied Physics, 2003, 93, 7, 3693- 3723.
28- K. Qi, F. Zasada, W. Piskorz, P. Indyka, J. Gryboś, M. Trochowski, M. Buchalska, M. Kobielusz, W. Macyk and Z. Sojka, SelfSensitized Photocatalytic Degradation of Colorless Organic Pollutants Attached to Rutile Nanorods-Experimental and Theoretical DFT + D Studies, The Journal of Physical Chemistry C, 2016, 120, 5442-5456.

29- Y. Yang, Z.-G. Zhang, H. Bin, et al. Side-Chain Isomerization on an n-type Organic Semiconductor ITIC Acceptor Makes 11.77\% High Efficiency Polymer Solar Cells. J. Am. Chem. Soc., 2016, 138 (45), 15011-15018.

30- S.A. Hawks, B.Y. Finck, B.J. Schwartz. Theory of Current Transients in Planar Semiconductor Devices: Insights and Applications to Organic Solar Cells. Phys. Rev. Appl., 2015, 3 (4), 044014.

31- C. Janáky, K. Rajeshwar. The role of (photo)electrochemistry in the rational design of hybrid conducting polymer/semiconductor assemblies: From fundamental concepts to practical applications. Prog. Polym. Sci., 2015, 43, 96-135.

32- K. Qi, R. Selvaraj, T. Al Fahdi, S. Al-Kindy, Y. Kim, G.-C. Wang, C.-W. Tai, M. Sillanpää, Enhanced photocatalytic activity of anatase-TiO2 nanoparticles by fullerene modification: A theoretical and experimental study, Applied Surface Science, 2016, 387, 750-758.

33- A. Facchetti. $\pi$-Conjugated Polymers for Organic Electronics and Photovoltaic Cell Applications $\uparrow$. Chem. Mater., 2011, 23 (3), 733-758.

34- K. Qi, S.-y. Liu, R. Selvaraj, W. Wang, Z. Yan,Comparison of Pt and Ag as co-catalyst on g-C3N4 for improving photocatalytic activity: experimental and DFT studies, Desalination and Water Treatment, 2019, 153, 244-252.

35- R.H. Reuss, G.B. Raupp, B.E. Gnade. Special issue on advanced flexible electronics for sensing applications, Proc. IEEE, 2015, 103 (4), 491-496.

36- P.G.V. Sampaio, M.O.A. González. Photovoltaic solar energy: Conceptual framework. Renew. Sustain. Energy Rev., 2017, 74, 590-601.

37 - L. D. Pettit and S. Bruckenstein, The Thermodynamics of Ion Association in Solution. I. An Extension of the Denison-Ramsey Equations, Journal of the American Chemical Society, 1966, 88, 21, 4783-4789.

38- . L. D. Landau and E. M. Lifshitz, Course of Theoretical Physics. Volume 3, Quantum Mechanics. Non-relativistic Theory. Second edition, revised and enlarged, PERGAMON PRESS. London,,1965, pp. 55-587.

39- M. Born, On Quantum Mechanics II, Zeitschrift fuer Physik, 1926, 35, 557, 1-65. 